\title{
Attenuation of colonic inflammation by partial replacement of dietary linoleic acid with $\alpha$-linolenic acid in a rat model of inflammatory bowel disease
}

\author{
Anupama Tyagi ${ }^{1}$, Uday Kumar ${ }^{2}$, Suryam Reddy ${ }^{1}$, Vadakattu S. Santosh ${ }^{1}$, Saazida B. Mohammed ${ }^{1}$, \\ Nasreen Z. Ehtesham ${ }^{3}$ and Ahamed Ibrahim ${ }^{1 *}$ \\ ${ }^{1}$ Department of Lipid Chemistry, National Institute of Nutrition, Indian Council of Medical Research, Hyderabad, India \\ ${ }^{2}$ Department of Pathology, National Institute of Nutrition, Indian Council of Medical Research, Hyderabad, India \\ ${ }^{3}$ Department of Molecular Biology, National Institute of Nutrition, Indian Council of Medical Research, Hyderabad, India
}

(Submitted 5 September 2011 - Final revision received 25 November 2011 - Accepted 28 November 2011 - First published online 16 January 2012)

\section{Abstract}

Increasing prevalence of inflammatory bowel disease may be due to imbalance in the intake of $n-6$ and $n-3$ PUFA in the diet. This study investigates the impact of varying ratios of dietary linoleic acid (LA, 18:2n-6) to $\alpha$-linolenic acid (ALA, 18:3n-3) on the inflammatory response in dextran sulphate sodium (DSS)-induced colitis. Weanling male Sprague-Dawley rats were divided into five groups: a non-colitic group with a LA:ALA ratio of 215 (CON-215), and colitic groups with LA:ALA ratios of 215 (DSS-215), 50 (DSS-50), 10 (DSS-10) and 2 (DSS-2). Blends of groundnut, palmolein and linseed oils were used to provide varying LA:ALA ratios. All the rats were fed the respective experimental isoenergetic diets containing $10 \%$ fat for $90 \mathrm{~d}$ and DSS was administered during the last $11 \mathrm{~d}$. Colonic inflammation was evaluated by clinical, biochemical and histological parameters. The results showed attenuation of colitis in the DSS- 2 group as evidenced by significant reductions in disease activity index, mucosal myeloperoxidase activity $(P<0 \cdot 05)$, alkaline phosphatase activity $(P<0 \cdot 01)$ and increase in colon length $(P<0 \cdot 01)$ compared to the groups fed with higher ratios (DSS-215). This was accompanied by significant reductions in mucosal proinflammatory cytokines TNF- $\alpha(P<0 \cdot 01)$ and IL-1 $\beta(P<0 \cdot 01)$ and improvement in the histological score. Further, ALA supplementation increased long-chain (LC) $n$-3 PUFA and decreased LC $n$-6 PUFA in colon structural lipids. These data suggest that substitution of one-third of LA with ALA (LA:ALA ratio 2) mitigates experimental colitis by down-regulating proinflammatory mediators.

Key words: Inflammatory bowel disease: Ulcerative colitis: Dextran sulphate sodium: Colon: Fatty acid composition: Linoleic acid: $\alpha$-linolenic acid ratio: Proinflammatory cytokines

Ulcerative colitis (UC) and Crohn's disease, the two types of inflammatory bowel disease (IBD), are characterised by recurrent episodes of inflammation and tissue degeneration ${ }^{(1)}$. Although the aetiology of IBD remains unknown, it has been suggested that it may be due to complex interactions among genetic, immune and environmental factors ${ }^{(2)}$. Over the past few decades, rapid Westernisation and altered lifestyle have led to a significant rise in the incidence of IBD in developing countries, including India ${ }^{(3)}$. Further, South Asian immigrants in the UK who had adopted Western lifestyle and dietary patterns reported increased risk of IBD $^{(4)}$. These observations are partially attributed to the role of dietary factors in the development of IBD. The current treatment with antiinflammatory drugs results in serious side effects, and therefore nutrition therapy is considered to be the best long-term option for disease management ${ }^{(5)}$. Among the dietary factors, both quantity and quality of dietary fat are being increasingly recognised as important modulators of the IBD risk ${ }^{(5,6)}$.

The PUFA linoleic acid (LA) and $\alpha$-linolenic acid (ALA) are the short-chain dietary precursors that are converted into biologically active long-chain (LC) PUFA such as arachidonic acid (20:4n-6), and EPA $(20: 5 n-3)$ and DHA (22:6n-3), respectively. The $n-6$ and $n-3$ PUFA are not interconvertible and are metabolically and functionally distinct ${ }^{(7)}$. The eicosanoids derived from $n$ - 6 PUFA are proinflammatory, whereas those derived from $n$-3 PUFA are anti-inflammatory. Moreover, $n-3$ PUFA down-regulate the proinflammatory genes. They are important components of cell membranes wherein they alter a number of cellular functions such as membrane-bound enzyme activities, transport, hormone binding and signal

Abbreviations: ALA, $\alpha$-linolenic acid; CON-215, non-colitic group with linoleic acid: $\alpha$-linolenic acid ratio of 215; DAI, disease activity index; DSS, dextran sulphate sodium; DSS-2, colitic group with linoleic acid: $\alpha$-linolenic acid ratio of 2; DSS-10, colitic group with linoleic acid: $\alpha$-linolenic acid ratio of 10 ; DSS-50, colitic group with linoleic acid: $\alpha$-linolenic acid ratio of 50; DSS-215, colitic group with linoleic acid: $\alpha$-linolenic acid ratio of 215 ; IBD, inflammatory bowel disease; LA, linoleic acid; LC, long-chain; MPO, myeloperoxidase; SCD-1, stearoyl coenzyme A desaturase-1; UC, ulcerative colitis. 
transduction $^{(8)}$. The PUFA composition of the cell membrane is determined by the dietary levels of $n-6$ and $n-3$ PUFA and their ratio. High intake of $n-6$ PUFA creates a proinflammatory environment, which in turn may affect the development or progression of several diet-related chronic diseases including $\operatorname{IBD}^{(9)}$. Over the past few decades, the intake of $n-6$ PUFA has shown an increase due to an increased consumption of LA-rich vegetable oils, whereas the consumption of $n-3$ PUFA has reduced. This resulted in a shift of the $n-6: n-3$ ratio to $>10: 1$ from the traditional $2: 1^{(10)}$. Epidemiological studies also have revealed that increased consumption of $n$-6 PUFA is associated with the risk of developing UC, while $n$-3 PUFA have been reported to confer protection ${ }^{(11,12)}$. A recent prospective cohort study also demonstrated that high levels of adipose tissue arachidonic acid, which reflect the dietary intake of $n-6$ PUFA, are associated with UC, thereby implicating the role of $n-6$ PUFA in the pathogenesis of $\mathrm{UC}^{(13)}$. Several intervention studies ${ }^{(14)}$ have documented the beneficial effects of marine $n-3$ PUFA in patients with IBD. However, the role of ALA has not been extensively investigated. The effects of increasing ALA and decreasing LA (varying the LA:ALA ratio) on inflammatory response in IBD have not been studied earlier. The present study attempts to investigate the effects of substituting dietary LA with ALA on colonic inflammation in the well-established dextran sulphate sodium (DSS)-induced model of UC by keeping total PUFA, MUFA and SFA constant.

\section{Material and methods}

\section{Animals and diets}

The study was approved by the institutional animal ethical committee of the National Institute of Nutrition, Hyderabad, India and all procedures involved in the animal experiments were performed in accordance with the guidelines of the committee for the purpose of control and supervision of experiments on animals (CPCSEA).

A total of seventy conventional male Sprague-Dawley weanling rats were obtained from the animal house facility of the National Institute of Nutrition (Hyderabad, India). The animals were housed individually in polypropylene cages and maintained under conventional housing conditions $\left(21 \pm 1{ }^{\circ} \mathrm{C}\right.$ and $12 \mathrm{~h}$ light $-12 \mathrm{~h}$ dark cycle). The rats were allowed free access to food and drinking-water. After habituation to commercial rat chow for $7 \mathrm{~d}$, the animals were randomly divided into five groups: a non-colitic control group with a LA:ALA ratio of 215 (CON-215; $n$ 14) and DSS-induced colitic groups with varying LA:ALA ratios of 215 (DSS-215; $n$ 14), 50 (DSS-50; $n$ 14), 10 (DSS-10; $n$ 14) and 2 (DSS-2; $n$ 14). The diet composition (g/100g diet) was as follows: starch $54.5 \%$, casein $25 \%$, fat $10 \%$, cellulose 5\%, salt mixture $4 \%$, vitamin mixture $1 \%$, L-cystine $0.3 \%$ and choline chloride $0 \cdot 2 \%$. The salt and vitamin mixtures were prepared according to AIN-93 ${ }^{(15)}$. Vegetable oil formulations were made by mixing groundnut oil, palmolein and linseed oil and used as a source of dietary fat. These diets have been used before as dietary ALA supplementation in different animal models ${ }^{(16)}$.
Table 1. Fatty acid (FA) composition of the diets ( $\mathrm{g} / 100 \mathrm{~g}$ diet)

\begin{tabular}{|c|c|c|c|c|c|}
\hline FA & CON-215 & DSS-215 & DSS-50 & DSS-10 & DSS-2 \\
\hline $16: 0$ & $2 \cdot 4$ & $2 \cdot 4$ & $2 \cdot 3$ & $2 \cdot 4$ & $2 \cdot 6$ \\
\hline $18: 0$ & 0.4 & 0.4 & 0.4 & 0.4 & 0.4 \\
\hline $20: 0$ & 0.1 & 0.1 & 0.1 & 0.1 & 0.1 \\
\hline ¿SFA & $2 \cdot 9$ & 2.9 & $2 \cdot 8$ & 2.9 & 3.1 \\
\hline ¿MUFA & 4.2 & 4.2 & $4 \cdot 3$ & $4 \cdot 3$ & 3.8 \\
\hline $18: 2 n-6$ & $2 \cdot 8$ & $2 \cdot 8$ & $2 \cdot 8$ & $2 \cdot 6$ & $2 \cdot 0$ \\
\hline $18: 3 n-3$ & 0.013 & 0.013 & 0.055 & 0.26 & 1.0 \\
\hline ¿PUFA & $2 \cdot 813$ & $2 \cdot 813$ & 2.9 & 2.9 & 3.0 \\
\hline$n-6: n-3$ & 215 & 215 & 50 & 10 & 2 \\
\hline PUFA:SFA & 1 & 1 & 1 & 1 & 1 \\
\hline
\end{tabular}

CON-215, non-colitic group with linoleic acid (LA): $\alpha$-linolenic acid (ALA) ratio of 215; DSS-215, colitic group with LA:ALA ratio of 215; DSS-50, colitic group with LA:ALA ratio of 50; DSS-10, colitic group with LA:ALA ratio of 10; DSS-2, colitic group with LA:ALA ratio of 2 .

The fatty acid composition of the dietary fats was determined by GC (Table 1) as described earlier ${ }^{(17)}$. Total PUFA (LA + ALA), SFA and MUFA were similar in all the groups. The LA:ALA ratio was altered by substituting LA with ALA to obtain the ratios of $215,50,10$ and 2 . All the animals were fed the respective experimental diets for $90 \mathrm{~d}$.

\section{Induction and assessment of colitis}

After $78 \mathrm{~d}$ of pre-feeding with specified experimental diets, UC was induced by oral administration of $4 \%$ DSS (molecular weight $36-50 \mathrm{kDa}$; MP Biomedicals) in sterilised drinkingwater for $11 \mathrm{~d}$ as described ${ }^{(18)}$, whereas the non-colitic group received plain water. All the animals were continued on the respective diets until they were killed. DSS solution was freshly prepared. Body weight, stool consistency and presence of faecal occult blood (detected by Hemospot kit; Coral Clinical Systems) or gross rectal bleeding were recorded daily for each animal. These parameters were used to calculate an average daily disease activity index (DAI) according to the criteria proposed by Cooper et al. ${ }^{(19)}$. Food and water intake were measured daily. After $11 \mathrm{~d}$ of induction of colitis, all the animals were fasted overnight. Blood was collected from the retro-orbital sinus in EDTA tubes and plasma was separated and stored at $-80^{\circ} \mathrm{C}$ for further analysis. Rats were euthanised by $\mathrm{CO}_{2}$ asphyxia. The abdominal cavity was exposed by midline laparotomy and the entire colon from the colocecal junction to the anal verge was removed. The length of the colon was measured. The colon was then flushed with icecold saline, opened longitudinally and a portion of the distal colon was immediately fixed in $10 \%$ neutral buffered formalin and embedded in paraffin for histopathological analysis. Mucosa was gently scraped with a microscopic slide and the collected mucosal samples were snap-frozen in liquid $\mathrm{N}_{2}$ and stored at $-80^{\circ} \mathrm{C}$ for the biochemical determinations.

\section{Measurement of colon myeloperoxidase and alkaline phosphatase activities}

Myeloperoxidase activity, a marker of neutrophil infiltration into the colon, was measured as described ${ }^{(20)}$. Briefly, approximately $50 \mathrm{mg}$ of freshly thawed mucosa was homogenised in 
$4 \mathrm{ml}$ of ice-cold $50 \mathrm{~mm}$-phosphate buffer (pH 6.0). The homogenate was centrifuged at $30000 \mathrm{~g}$ for $30 \mathrm{~min}$ at $4^{\circ} \mathrm{C}$. The resulting supernatant was discarded and the pellet was re-suspended in phosphate buffer and centrifuged again as previously described. This process was repeated three times to remove $\mathrm{Hb}$ and other blood products which are known to interfere with the assay. The final pellet was solubilised in ten volumes of ice-cold $50 \mathrm{~mm}$-phosphate buffer containing $0.5 \%$ hexadecyltrimethyl ammonium bromide and sonicated on ice to solubilise the enzyme. The sonicated extract was allowed to stand at $4^{\circ} \mathrm{C}$ for $20 \mathrm{~min}$ and then centrifuged at $12000 \mathrm{~g}$ for $15 \mathrm{~min}$ at $4^{\circ} \mathrm{C}$. Myeloperoxidase (MPO) activity in the supernatant was assayed by mixing $0.1 \mathrm{ml}$ of the supernatant with $2.9 \mathrm{ml}$ of $50 \mathrm{~mm}$-phosphate buffer ( $\mathrm{pH}$ 6.0) containing $0.167 \mathrm{mg} / \mathrm{ml}$ of $\mathrm{O}$-dianisidine and $0.0005 \% \mathrm{H}_{2} \mathrm{O}_{2}$. The change in absorbance was recorded at $460 \mathrm{~nm}$ over $3 \mathrm{~min}$. MPO activity was expressed as units/g tissue, where 1 unit corresponds to the activity required to degrade $1 \mu \mathrm{mol} \mathrm{H}_{2} \mathrm{O}_{2}$ in $1 \mathrm{~min}$ at $25^{\circ} \mathrm{C}$.

Alkaline phosphatase activity, a marker of colonic inflammation was measured using disodium $p$-nitrophenol phosphate as the substrate ${ }^{(21)}$. Here, 1 unit of alkaline phosphatase activity is the enzyme activity which hydrolyses $1 \mu \mathrm{mol}$ of substrate in $1 \mathrm{~min}$ at $37^{\circ} \mathrm{C}$, and the activity is expressed as $\mathrm{mU} / \mathrm{mg}$ protein. Protein was estimated by Lowry's method ${ }^{(22)}$.

\section{Lipid extraction and quantification of colon fatty acids}

Total lipids were extracted from colonic mucosa by the Folch $\operatorname{method}^{(23)}$. Neutral lipids were separated from phospholipids by TLC on silica gel $G$ with hexane-diethyl ether-acetic acid (80:20:1, v/v). The fatty acid profile of phospholipids was determined after methylation ${ }^{(17)}$. The phospholipid fraction was trans-esterified by methanolysis $\left(2 \% \mathrm{H}_{2} \mathrm{SO}_{4}\right.$ in methanol) for $4 \mathrm{~h}$ at $70^{\circ} \mathrm{C}$. After cooling, the resulting fatty acid methyl esters were extracted with petroleum ether and transferred to gas chromatograph vials. Fatty acid methyl esters were separated and quantified with an Agilent gas chromatograph (Agilent 6890; Agilent Technologies) using an SP-2330 capillary column $(30 \mathrm{~m} \times 0.3 \mathrm{~mm}$ inner diameter; Supelco) equipped with a flame ionisation detector. Fatty acid methyl esters were identified by comparison of retention times with authentic standards (Nuchek Prep).

\section{Determination of nitrite and nitrate $\left(\mathrm{NO}_{\mathrm{x}}\right)$ and lipid peroxide levels}

Plasma and colonic mucosal content of nitrite and nitrate $\left(\mathrm{NO}_{x}\right)$ were estimated according to the method of Miranda et $a l .{ }^{(24)}$. Nitrate was reduced to nitrite using vanadium trichloride and the nitrite was measured by the Griess reaction. Results are expressed as $\mathrm{NO}_{x} / \mathrm{mg}$ protein. Lipid peroxidation was assayed by measuring the amount of thiobarbituric acidreactive substances. Plasma and colonic mucosal thiobarbituric acid-reactive substances were estimated as described ${ }^{(25)}$.

\section{Determination of colonic mucosal cytokine levels}

Colonic mucosa was homogenised in $10 \mathrm{~mm}$-phosphate buffer saline (pH 7.4) containing 1 mm-EDTA, 5 mm-dithiothreitol and $10 \mu \mathrm{g} / \mathrm{ml}$ each of aprotanin, leupeptin and pepstatin A using a polytron homogeniser. The homogenate was centrifuged at $10000 \mathrm{~g}$ for $5 \mathrm{~min}$ at $4^{\circ} \mathrm{C}$. The supernatant was processed for ELISA using commercial kits (Quantikine colorimetric sandwich ELISA; R\&D Systems). The content of TNF- $\alpha$ and IL-1 $\beta$ was determined according to the manufacturer's protocol. The results were expressed as concentration per mg protein.

\section{Histological assessment of colonic inflammation}

The extent of tissue damage was assessed microscopically using a semi-quantitative scoring system as described previously ${ }^{(26)}$. Distal colon was fixed in $10 \%$ formalin, dehydrated and paraffin embedded. Sections $(4 \mu \mathrm{m})$ were stained with haematoxylin and eosin and scored based on the parameters such as severity of inflammation (0-3: none, slight, moderate and severe), extent of injury ( $0-3$ : none, mucosal, mucosal and submucosal, transmural) and crypt damage ( $0-4$ : none, basal 1/3 damaged, basal 2/3 damaged, entire crypt damaged and epithelium lost). Total score (maximum score $=40$ ) was obtained by multiplying each parameter by a factor reflecting the percentage of tissue involved $\left(x_{1}: 0-25 \%, x_{2}: 26-50 \%, x_{3}: 51-75 \%, x_{4}\right.$ : $76-100 \%)$ and all numbers were summed.

\section{Statistical analysis}

Statistical analysis was done using the SPSS (version 10; SPSS, Inc.) statistical program. The results are expressed as means

Table 2. Effect of varying dietary linoleic acid (LA): $\alpha$-linolenic acid (ALA) ratios on water intake, food intake and body weight changes in dextran sulphate sodium (DSS)-induced colitis

(Mean values with their standard errors, $n$ 14)

\begin{tabular}{|c|c|c|c|c|c|c|c|c|c|c|}
\hline & \multicolumn{2}{|c|}{ CON-215 } & \multicolumn{2}{|c|}{ DSS-215 } & \multicolumn{2}{|c|}{ DSS-50 } & \multicolumn{2}{|c|}{ DSS-10 } & \multicolumn{2}{|c|}{ DSS-2 } \\
\hline & Mean & SE & Mean & SE & Mean & SE & Mean & SE & Mean & SE \\
\hline Water intake $(\mathrm{ml} / \mathrm{d})^{*}$ & $19 \cdot 0^{\mathrm{a}}$ & 0.5 & $22 \cdot 0^{\mathrm{b}}$ & 0.8 & $22 \cdot 5^{\mathrm{b}}$ & $1 \cdot 3$ & $22 \cdot 5^{\mathrm{b}}$ & $1 \cdot 2$ & $23.0^{\mathrm{b}}$ & 1.3 \\
\hline Food intake $(\mathrm{g} / \mathrm{d})^{*}$ & $17 \cdot 2^{\mathrm{b}}$ & 0.4 & $14 \cdot 4^{a}$ & 0.6 & $14 \cdot 1^{\mathrm{a}}$ & 1.0 & $14 \cdot 0^{\mathrm{a}}$ & 0.6 & $16 \cdot 0^{a, b}$ & 0.6 \\
\hline Body weight change at day $11(\%) \dagger$ & $103 \cdot 3^{\mathrm{b}}$ & 0.6 & $96 \cdot 1^{a}$ & $2 \cdot 0$ & $95 \cdot 0^{\mathrm{a}}$ & $2 \cdot 1$ & $96 \cdot 0^{\mathrm{a}}$ & 1.0 & $98 \cdot 0^{\mathrm{a}}$ & 1.0 \\
\hline
\end{tabular}

CON-215, non-colitic group with LA: ALA ratio of 215; DSS-215, colitic group with LA:ALA ratio of 215; DSS-50, colitic group with LA:ALA ratio of 50;

DSS-10, colitic group with LA:ALA ratio of 10; DSS-2, colitic group with LA:ALA ratio of 2.

a,b Mean values within a row with unlike superscript letters were significantly different $(P<0.05)$

* Water and food intake corresponds to the period $(0-11 \mathrm{~d})$ during DSS treatment.

†Percentage indicates body weight changes at day 11 compared with day 0 . 


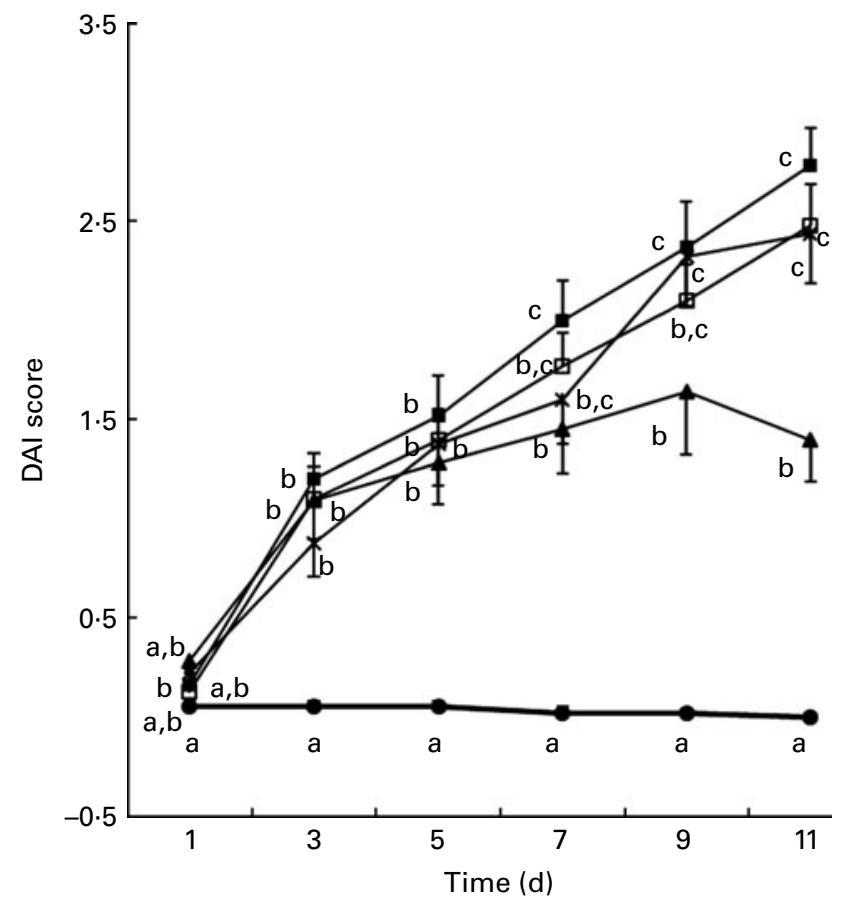

Fig. 1. Time course of changes in the disease activity index (DAI) in non-colitic rats fed a diet with a linoleic acid (LA): $\alpha$-linolenic acid (ALA) ratio of 215 $(--)$ and dextran sulphate sodium-induced colitic rats fed diets with a LA:ALA ratio of $215(\square), 50(-)), 10(\rightarrow)$ or $2(\neg-)$. Values are means, with standard errors represented by vertical bars $\left(n\right.$ 14). ${ }^{\mathrm{a}, \mathrm{b}, \mathrm{c}}$ Mean values at a time with unlike letters were significantly different $(P<0.05)$.

(A)
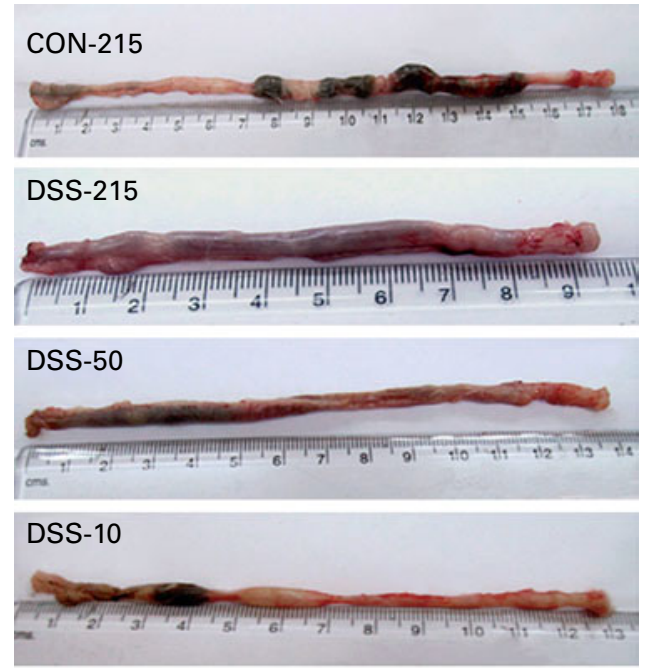

DSS-2

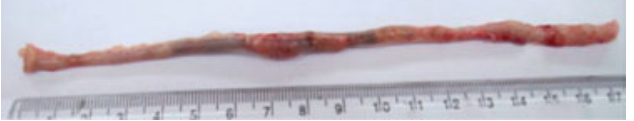

with their standard errors. One-way ANOVA was used to test for significant difference between the dietary groups. Post boc comparisons were performed using the least significant difference test. A value of $P<0.05$ was considered statistically significant.

\section{Results}

\section{Food intake, water intake and body weight changes}

There was no difference in food intake and body weight gain among the groups before the induction of colitis (data not shown). Table 2 describes the general characteristics of the experimental groups after the DSS-induced colitis. DSS administration resulted in significant decreases in food intake and body weight gain. The decrease in body weight gain could be attributed to diarrhoea which is a common symptom of UC. Substitution of LA with ALA (LA:ALA ratio of 2) increased the food intake without any significant change in body weight. Compared to the non-colitic control group, water consumption was higher in the colitic groups. However, there was no difference in the consumption of water in the colitic groups.

\section{Dietary increase in $\alpha$-linolenic acid and concomitant decrease in linoleic acid attenuated clinical symptoms and shortening of colon in dextran sulphate sodium-induced colitis}

Rats given $4 \%$ DSS in drinking-water for $11 \mathrm{~d}$ developed symptoms of colitis without mortality. In comparison to noncolitic controls, DSS-administered rats developed loose stools after the 2nd or 3rd day, which turned into diarrhoea on the

(B)

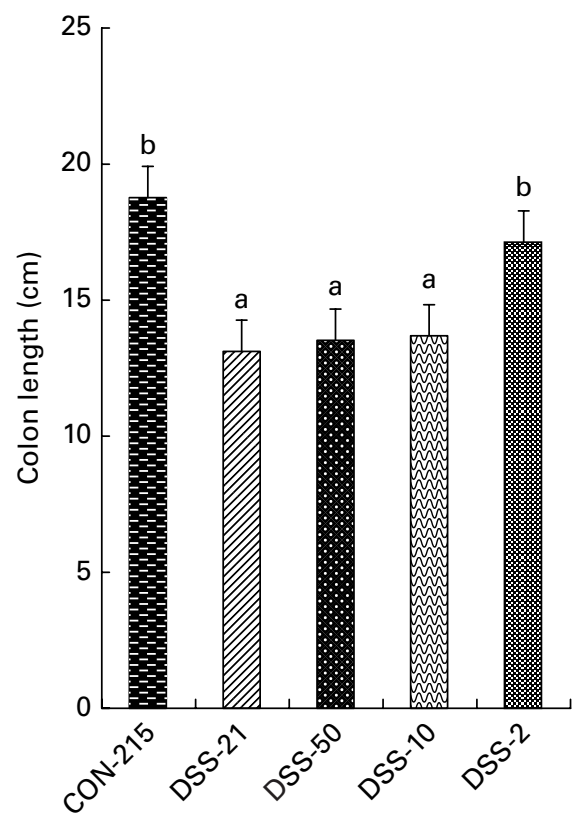

Fig. 2. (A) Macroscopic view of the colon showing changes in length in rats fed a diet with a linoleic acid (LA): $\alpha$-linolenic acid (ALA) ratio of $215(C O N-215)$ and dextran sulphate sodium (DSS)-induced colitic rats fed diets with a LA:ALA ratio of 215 (DSS-215), 50 (DSS-50), 10 (DSS-10) or 2 (DSS-2). (B) Colon length in $\mathrm{cm}$. Values are means, with standard errors represented by vertical bars $(n 14){ }^{\mathrm{a}, \mathrm{b}}$ Mean values with unlike letters were significantly different $(P<0 \cdot 05)$. 
5th day. By the 10th or 11th day, gross rectal bleeding was observed. Based on the clinical symptoms (weight loss, stool consistency and intestinal bleeding), the DAI which assesses the severity of colitis was calculated on a daily basis and the results are presented in Fig. 1. The DAI became progressive from the 2nd day after DSS treatment and peaked between days 9 and 11 depending upon the dietary treatment. Rats fed a diet with a LA:ALA ratio of 2 (DSS-2) had significantly less severity of the colitis as evidenced by a lower DAI score. The decrease in DAI score was apparent on days 9, 10 and 11 compared to other groups. The severity of the colitis was also assessed by measuring the length of the colon which is an indirect marker of inflammation. As shown in Fig. 2, DSS administration caused significant reduction in colon length in rats fed diets with high LA:ALA ratios (DSS-215, DSS-50 and DSS-10) compared with the non-colitic control rats (CON-215). However, decreasing the LA:ALA ratio to 2 (DSS-2) significantly increased the colon length and was comparable with the non-colitic control rats.

\section{Dietary $\alpha$-linolenic acid attenuated neutrophil infiltration and alkaline phosphatase activities in dextran sulphate} sodium-induced colitis

Inflammatory status of the colon was assessed biochemically by determining MPO activity, an enzymatic marker of neutrophil infiltration. DSS treatment significantly increased colonic MPO activity to a level approximately thirteen times higher than the non-colitic control group (Fig. 3). However, substitution of LA with ALA (DSS-2) significantly decreased the MPO activity, suggesting that ALA exerts an anti-inflammatory effect by reducing the neutrophil infiltration into the colonic mucosa. In addition to MPO activity, the degree of colonic inflammation was also assessed by measuring colonic alkaline phosphatase activity which has been proposed as a marker of colonic inflammation. DSS-induced colitis was associated with a significant increase in colonic alkaline phosphatase, and by increasing ALA in the diet (DSS-2), the activity was reduced.

\section{Colonic phospholipid fatty acid profile}

The phospholipid fatty acid composition of the colon, which reflects the fatty acid composition of the dietary fats, is shown in Table 3. DSS treatment, as such, did not have any effect on fatty acid composition. However, altering the dietary LA:ALA ratio in the DSS-treated groups showed marked effects on $n-6$ and $n-3$ PUFA composition. Decreasing the LA:ALA ratio in the diet was associated with a decrease in the proportion of LC $n-6$ PUFA (20:4n-6, 22:4n-6 and 22:5n-6) and an increase in LA. This was accompanied by a progressive increase in the proportion of ALA and LC $n-3$ PUFA (20:5n-3, $22: 5 n-3$ and $22: 6 n-3)$. Incorporation of EPA was observed in the group which was fed a diet having the lowest LA:ALA ratio (the highest ALA content). Remarkably, decreasing the LA:ALA ratio to 2 lowered the levels of stearic acid (18:0) and raised that of oleic acid $(18: 1 n-9)$, with consequent increase in the $18: 1 / 18: 0$ ratio, which is the estimated index of stearoyl coenzyme A desaturase-1 (SCD-1) activity.
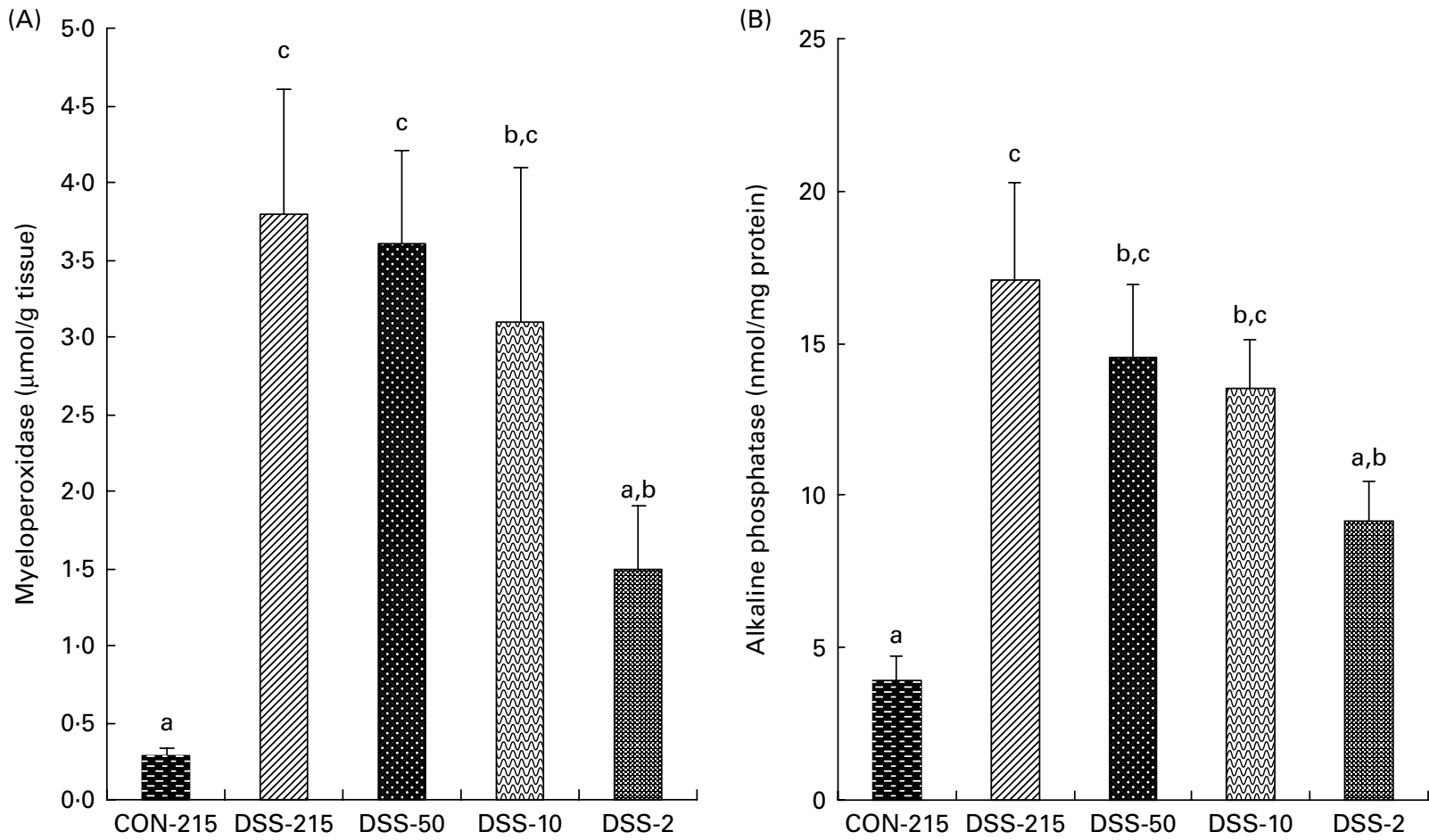

Fig. 3. (A) Colon myeloperoxidase and (B) alkaline phosphatase activities in non-colitic rats fed a diet with a linoleic acid (LA): $\alpha$-linolenic acid (ALA) ratio of 215 (CON-215) and dextran sulphate sodium (DSS)-induced colitic rats fed diets with a LA:ALA ratio of 215 (DSS-215), 50 (DSS-50), 10 (DSS-10) or 2 (DSS-2). Values are means, with standard errors represented by vertical bars $(n 8) .{ }^{a, b, c}$ Mean values with unlike letters were significantly different $(P<0.05)$. 
Table 3. Colon phospholipid fatty acid composition in non-colitic rats fed a diet with a linoleic acid (LA): $\alpha$-linolenic acid (ALA) ratio of 215 and dextran sulphate sodium (DSS)-induced colitic rats fed diets with a LA:ALA ratio of 215, 50, 10 or 2 (Mean values with their standard errors, $n 8$ )

\begin{tabular}{|c|c|c|c|c|c|c|c|c|c|c|}
\hline \multirow[b]{2}{*}{ Fatty acids (nmol \%) } & \multicolumn{2}{|c|}{ CON-215 } & \multicolumn{2}{|c|}{ DSS-215 } & \multicolumn{2}{|c|}{ DSS-50 } & \multicolumn{2}{|c|}{ DSS-10 } & \multicolumn{2}{|c|}{ DSS-2 } \\
\hline & Mean & SE & Mean & $\mathrm{SE}$ & Mean & SE & Mean & SE & Mean & SE \\
\hline $14: 0$ & $1 \cdot 2^{\mathrm{a}}$ & 0.2 & $1 \cdot 2^{\mathrm{a}}$ & 0.1 & $1 \cdot 1^{\mathrm{a}}$ & 0.1 & $1 \cdot 3^{\mathrm{a}}$ & 0.1 & $1 \cdot 1^{\mathrm{a}}$ & 0.1 \\
\hline $16: 0$ & $24 \cdot 3^{\mathrm{a}, \mathrm{b}}$ & 1.2 & $27 \cdot 1^{\mathrm{b}}$ & $1 \cdot 1$ & $25 \cdot 2^{a, b}$ & 1.3 & $25 \cdot 0^{a, b}$ & $2 \cdot 0$ & $21 \cdot 2^{\mathrm{a}}$ & $2 \cdot 0$ \\
\hline $16: 1$ & $2 \cdot 0^{\mathrm{a}}$ & 0.3 & $2 \cdot 0^{\mathrm{a}}$ & 0.1 & $2 \cdot 0^{a}$ & 0.1 & $2 \cdot 0^{\mathrm{a}}$ & 0.1 & $1.5^{\mathrm{a}}$ & 0.1 \\
\hline $18: 0$ & $18 \cdot 5^{\mathrm{b}}$ & $2 \cdot 0$ & $19 \cdot 2^{\mathrm{b}}$ & 1.0 & $19 \cdot 0^{\mathrm{b}}$ & 1.4 & $17 \cdot 0^{\mathrm{b}}$ & $2 \cdot 0$ & $11 \cdot 0^{\mathrm{a}}$ & 1.0 \\
\hline $18: 1$ & $20 \cdot 0^{\mathrm{a}}$ & 3.4 & $18 \cdot 4^{a}$ & 2.0 & $19 \cdot 3^{a}$ & 3.0 & $23.0^{\mathrm{a}}$ & 3.3 & $35.0^{\mathrm{b}}$ & 2.4 \\
\hline $18: 1 / 18: 0$ & $1 \cdot 2^{\mathrm{a}}$ & 0.3 & $1 \cdot 1^{a}$ & 0.2 & $1 \cdot 1^{\mathrm{a}}$ & 0.3 & $2 \cdot 0^{\mathrm{a}}$ & 0.4 & $3.3^{\mathrm{b}}$ & 0.5 \\
\hline $18: 2 n-6$ & $10 \cdot 4^{a}$ & 1.5 & $10.0^{\mathrm{a}}$ & 1.0 & $11.0^{\mathrm{a}}$ & 1.3 & $12 \cdot 3^{\mathrm{a}, \mathrm{b}}$ & 1.5 & $14 \cdot 4^{\mathrm{b}}$ & 1.1 \\
\hline $20: 4 n-6$ & $15 \cdot 0^{\mathrm{b}}$ & 2.0 & $14 \cdot 0^{\mathrm{b}}$ & 1.4 & $15 \cdot 2^{b}$ & 1.2 & $13 \cdot 0^{\mathrm{b}}$ & 1.3 & $8 \cdot 2^{a}$ & 1.0 \\
\hline $22: 4 n-6$ & $6 \cdot 2^{\mathrm{b}}$ & 1.0 & $6 \cdot 0^{\mathrm{b}}$ & 1.0 & $5 \cdot 5^{\mathrm{b}}$ & 0.5 & $5 \cdot 0^{\mathrm{b}}$ & 1.0 & $3 \cdot 0^{a}$ & 0.4 \\
\hline $22: 5 n-6$ & $2 \cdot 0^{c}$ & 0.1 & $2 \cdot 0^{c}$ & 0.1 & $1 \cdot 2^{\mathrm{b}}$ & 0.1 & $0.5^{\mathrm{a}}$ & 0.2 & ND & - \\
\hline $18: 3 n-3$ & ND & - & ND & - & ND & - & ND & - & $1 \cdot 3$ & 0.1 \\
\hline $20: 5 n-3$ & ND & - & ND & - & ND & - & ND & - & 1.5 & 0.1 \\
\hline $22: 5 n-3$ & ND & - & ND & - & 0.4 & 0.1 & 1.0 & 0.1 & 1.0 & 0.1 \\
\hline $22: 6 n-3$ & $1.0^{\mathrm{a}}$ & 0.1 & $1 \cdot 0^{\mathrm{a}}$ & 0.1 & $1.0^{\mathrm{a}}$ & 0.1 & $2 \cdot 0^{\mathrm{b}}$ & 0.2 & $3 \cdot 3^{c}$ & 0.3 \\
\hline$\sum$ LC $n-6$ PUFA* & $23 \cdot 0^{\mathrm{b}}$ & $2 \cdot 3$ & $21 \cdot 3^{\mathrm{b}}$ & $2 \cdot 0$ & $22 \cdot 0^{\mathrm{b}}$ & $2 \cdot 0$ & $18 \cdot 0^{\mathrm{b}}$ & 2.0 & $11 \cdot 1^{\mathrm{a}}$ & 1.3 \\
\hline$\Sigma L C n-3$ PUFA & $0.7^{\mathrm{a}}$ & 0.1 & $0.7^{\mathrm{a}}$ & 0.1 & $1 \cdot 3^{\mathrm{a}}$ & 0.1 & $2 \cdot 3^{\mathrm{b}}$ & 0.2 & $5 \cdot 5^{c}$ & 0.5 \\
\hline
\end{tabular}

CON-215, non-colitic group with LA:ALA ratio of 215; DSS-215, colitic group with LA:ALA ratio of 215; DSS-50, colitic group with LA:ALA ratio of 50; DSS-10, colitic group with LA:ALA ratio of 10; DSS-2, colitic group with LA:ALA ratio of 2; ND, non-detectable; LC, long-chain. ${ }^{a, b, c}$ Mean values within a row with unlike superscript letters were significantly different $(P<0.05)$.

${ }^{*} \Sigma$ LC $n-6$ PUFA $=20: 4 n-6+22: 4 n-6+22: 5 n-6$

$+\Sigma$ LC $n-3$ PUFA $=20: 5 n-3+22: 5 n-3+22: 6 n-3$

\section{Effects of dietary $\alpha$-linolenic acid on proinflammatory cytokines in colonic tissue}

It is known that TNF- $\alpha$ and IL-1 $\beta$ are the major inflammatory mediators in the pathogenesis of UC and they are produced from both inflammatory cells and mucosal epithelial cells during inflammation. As shown in Fig. 4, $11 \mathrm{~d}$ after colitis induction by DSS administration, the tissue levels of TNF- $\alpha$ and IL-1 $\beta$ were observed to be significantly higher than that of the non-colitic control rats. Decreasing the LA:ALA ratio to 2 significantly suppressed DSS-induced increase in TNF- $\alpha$ and IL-1 $\beta$ levels.

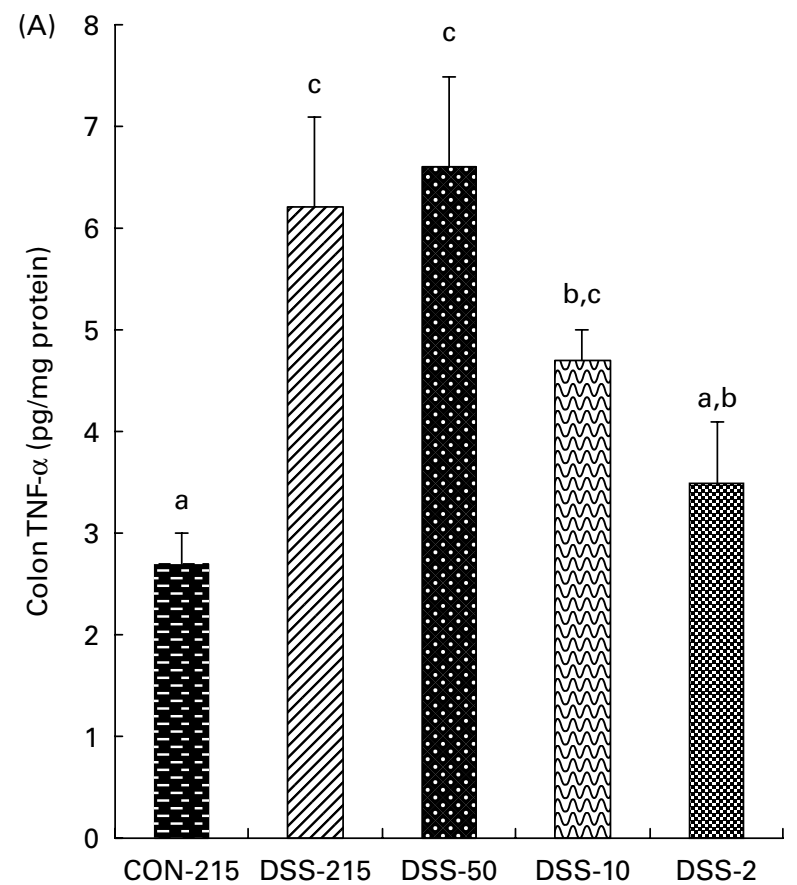

(B)

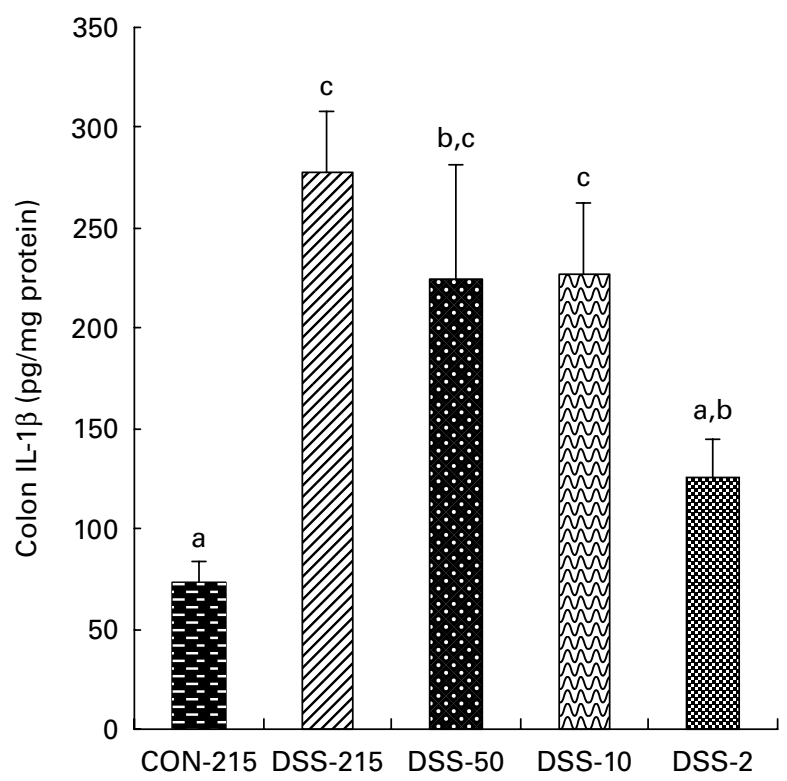

Fig. 4. (A) Colon TNF- $\alpha$ and (B) colon IL-1 $\beta$ levels in non-colitic rats fed a diet with a linoleic acid (LA): $\alpha$-linolenic acid (ALA) ratio of 215 (CON-215) and dextran sulphate sodium (DSS)-induced colitic rats fed diets with a LA:ALA ratio of 215 (DSS-215), 50 (DSS-50), 10 (DSS-10) or 2 (DSS-1). Values are means, with standard errors represented by vertical bars $(n 8)$. ${ }^{\text {a,b,c }}$ Mean values with unlike letters were significantly different $(P<0.05)$. 

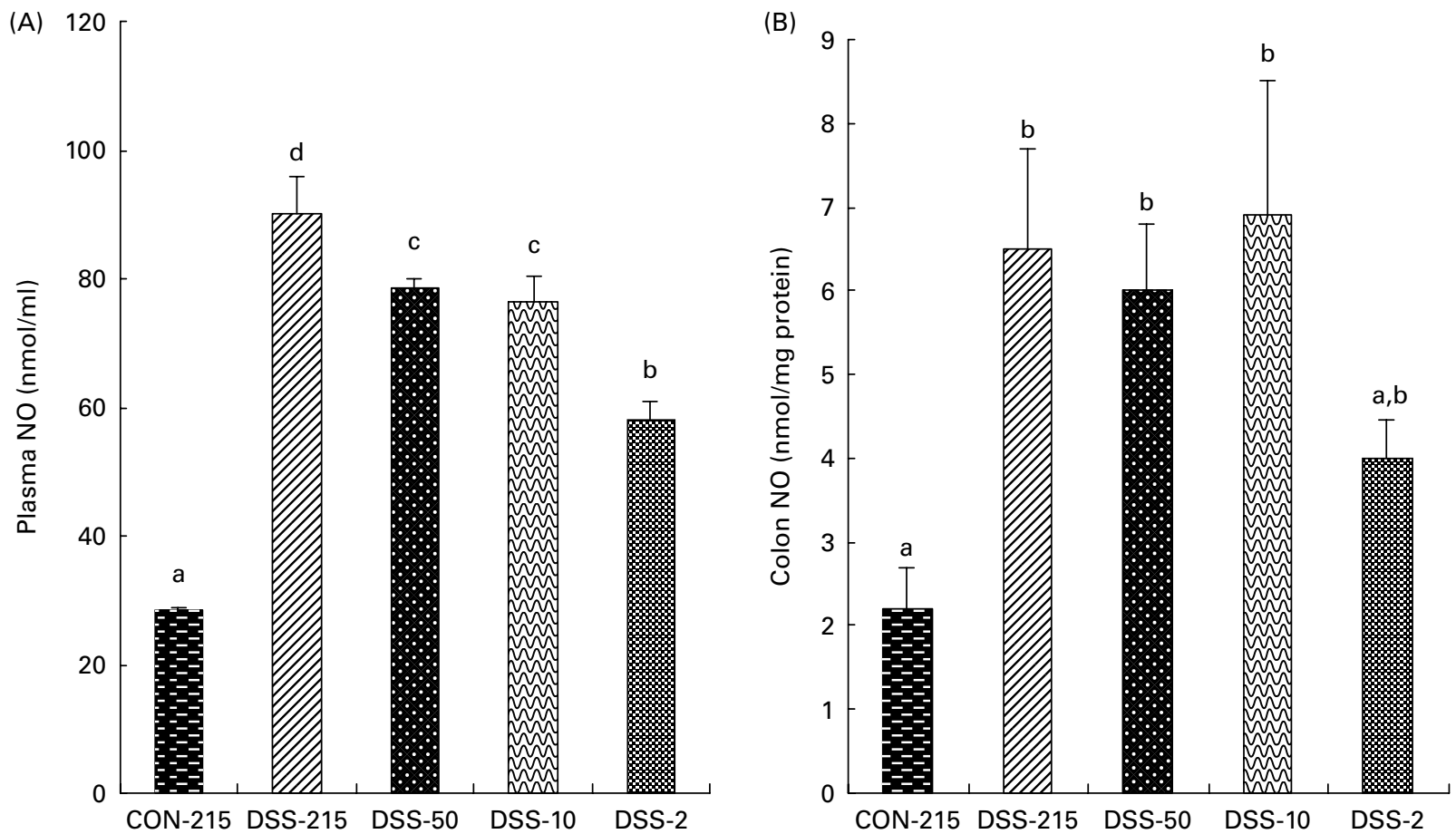

Fig. 5. (A) Plasma nitric oxide and (B) colon nitric oxide levels in non-colitic rats fed a diet with a linoleic acid (LA): $\alpha$-linolenic acid (ALA) ratio of 215 (CON-215) and dextran sulphate sodium (DSS)-induced colitic rats fed diets with a LA:ALA ratio of 215 (DSS-215), 50 (DSS-50), 10 (DSS-10) or 2 (DSS-2). Values are means, with standard errors represented by vertical bars $(n 8) .{ }^{a, b, c, d}$ Mean values with unlike letters were significantly different $(P<0.05)$.

\section{Effects of dietary $\alpha$-linolenic acid on plasma and colon} tissue thiobarbituric acid-reactive substances and $\mathrm{NO}_{\times}$

Reactive oxygen species and NO play a key role in the pathophysiology of IBD. Neither DSS treatment nor dietary ALA altered the plasma and colon thiobarbituric acid-reactive substance levels (data not shown). As shown in Fig. 5, DSS administration significantly increased plasma and colon $\mathrm{NO}_{x}$ levels. This increase in colon $\mathrm{NO}_{x}$ was completely prevented by ALA supplementation. Although ALA supplementation decreased plasma $\mathrm{NO}_{x}$, it did not normalise the levels.

\section{Histopathological changes}

Colonic inflammation was also evaluated by histopathological analysis using haematoxylin-eosin-stained sections of the distal colon. As shown in Fig. 6, no histological abnormalities were observed in non-colitic rats. In contrast, DSS administration showed typical changes in colonic architecture with mucosal ulceration, massive infiltration of inflammatory cells into mucosa and submucosa, depletion of goblet cells, erosion and crypt loss. The histological score which reflects overall mucosal damage and infiltration of inflammatory cells was increased after DSS administration. A reduction in the histological score was observed in rats which were fed a diet with a LA:ALA ratio of 2 . This was characterised by increased epithelial regeneration and decreased infiltration of inflammatory cells.

\section{Discussion}

DSS-induced colitis is a well-characterised experimental model of UC and exhibits several morphological and pathophysiological features that resemble human $\mathrm{UC}^{(27)}$. Although the exact mechanism of colitis induction is not elucidated, it is believed that destruction of the epithelial barrier by DSS leads to phagocytosis of lamina propia cells and production of cytokines, thereby triggering the inflammatory response. The results presented in this study demonstrate that rats which were fed a diet with a high LA:ALA ratio and subjected to DSS administration exhibit increased colonic inflammation. This is evident by their increased DAI and histology scores, increased infiltration of inflammatory cells and production of proinflammatory cytokines in colonic mucosa. In contrast, the substitution of one-third of dietary LA with ALA (LA:ALA ratio of 2) resulted in significant protection from inflammatory damage.

Several studies ${ }^{(28-31)}$ have demonstrated the protective effects of LC $n-3$ PUFA in various animal models of IBD. Further, transgenic mice engineered to express fat 1 gene encoding an $n$-3 fatty acid desaturase, which is capable of converting $n$-6 PUFA to LC $n$-3 PUFA, showed protection against DSS-induced colitis ${ }^{(32)}$. Although there are several studies on the anti-inflammatory potential of LC $n$-3 PUFA, the role of ALA in inflammation is yet to be established. ALA undergoes a series of complicated desaturation and chain elongation pathways to become converted into biologically active LC $n$-3 PUFA. This is influenced by several factors including sex, genetic factors, physiological status and dietary 
(A)
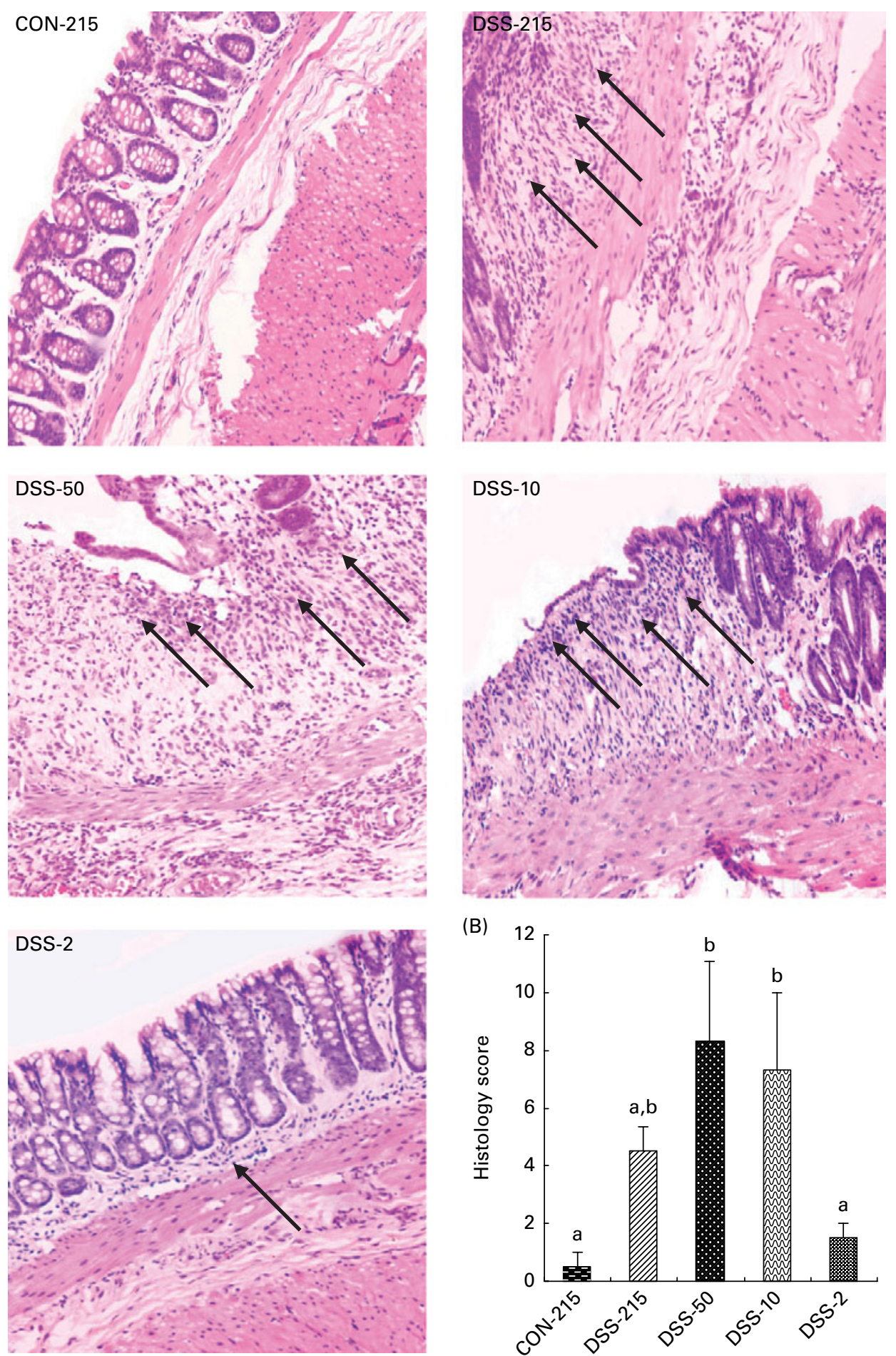

Fig. 6. Colon histology and histology score in rats fed diets with varying linoleic acid (LA): $\alpha$-linolenic acid (ALA) ratios and subjected to dextran sulphate sodium (DSS)-induced colitis. (A) Photomicrograph illustrates haematoxylin-eosin-stained sections of distal colon (original magnification $200 \times$ ) of rats fed diets with a LA:ALA ratio of $215,50,10$ or 2. The colons from non-colitic rats showed normal histology (CON-215). Rats fed diets with a LA:ALA ratio of 215 (DSS-215), 50 (DSS-50) or 10 (DSS-10) and subjected to DSS treatment showed severe mucosal damage characterised by a loss of crypts with a predominant infiltration of inflammatory cells into both mucosa and submucosa. Rats fed a diet with a LA:ALA ratio of 2 showed reduced inflammation and restoration of mucosal tissue. Infiltration of inflammatory cells is indicated by black arrows. (B) Histogram depicts the histology scores from non-colitic rats and DSS-treated colitic rats. DSS treatment increased the histology score. Decreasing the LA:ALA ratio to 2 decreased the histology score. Values are means, with standard errors represented by vertical bars $(n 4) .{ }^{\mathrm{a}, \mathrm{b}}$ Mean values with unlike letters were significantly different $(P<0.05)$. 
factors $^{(33)}$. High intake of LA and hence a high LA:ALA ratio could inhibit the conversion of ALA, which is supported by several animal studies ${ }^{(34-36)}$. However, recent human studies using a tracer isotope technique demonstrated that the conversion of ALA to LC $n$ - 3 PUFA is determined by the absolute levels of LA and ALA and not the ratio ${ }^{(37)}$. These studies suggest that decreasing LA together with an increase in ALA would be the most appropriate way of optimising the conversion of ALA to LC $n-3$ PUFA. Therefore, in the present study, the impact of ALA on DSS-induced UC was investigated by substituting LA with ALA and by keeping total PUFA constant.

Altered fatty acid metabolism has been demonstrated in patients with $\operatorname{IBD}^{(38)}$. In the present study, compared to the non-colitic group, DSS treatment did not alter the colonic mucosal fatty acid composition (CON-215 v. DSS-215). However, substituting varying levels of LA with ALA in DSS-treated groups increased LC $n-3$ PUFA and decreased the levels of LC $n$-6 PUFA. Studies in rats ${ }^{(39)}$ and piglets ${ }^{(40)}$ showed that maximum DHA accumulation in various tissues occurs at a ratio of 1:1 or 2:1, respectively. However, in these studies, LA:ALA ratio was altered by increasing ALA and keeping LA levels in the diet constant. The results of the present study are in agreement with our earlier studies in rats which demonstrated that maximum incorporation of DHA in diaphragm phospholipids occurs at the LA:ALA ratio of $2^{(16)}$

The observed reduction in inflammatory response by ALA supplementation could possibly be due to a marked reduction in arachidonic acid and an increase in EPA and DHA levels in the colon. It is generally believed that $n-6$ and n-3 PUFA are having opposing effects on inflammatory response ${ }^{(41)}$. This is due to the fact that the eicosanoids generated from n-6 PUFA are proinflammatory. They recruit neutrophils into the site of injury and elicit the inflammatory response. High levels of these proinflammatory eicosanoids have been reported in the colonic mucosa of patients with IBD and animal models of colitis ${ }^{(29,31,42,43)}$. On the contrary, $n-3$ PUFA inhibit the production of these proinflammatory eicosanoids. Moreover, the eicosanoids formed from EPA are less inflammatory. Recently, several studies reported that EPA and DHA generate novel anti-inflammatory bioactive molecules belonging to the resolvin and protectin family which play a major role in resolving inflammation ${ }^{(44,45)}$. Although we did not measure the eicosanoid levels in the present study, the protective effect of ALA supplementation could be due to a shift in arachidonic acid-derived mediators to EPAand DHA-derived mediators. Intriguingly, the data on colon phospholipid fatty acid composition showed that substitution of LA with ALA increased the stearic acid:oleic acid ratio, which is an index of SCD-1 activity. There are a few studies which investigated the role of SCD-1 in inflammation. SCD-1 deficiency has been shown to cause chronic inflammation of skin ${ }^{(46)}$, increase in the systemic markers of inflammation and susceptibility to atherosclerosis ${ }^{(47)}$. Chen et al. ${ }^{(48)}$ demonstrated that SCD-1 deficiency exacerbates DSS-induced colitis, whereas oleic acid supplementation or SCD-1 overexpression prevents the DSS-induced colitis, suggesting that SCD-1 may play a major role in colonic inflammation. Nevertheless, subsequent studies failed to demonstrate the role of SCD-1 in
DSS-induced colitis ${ }^{(49)}$. Further studies are required to explore the role of SCD-1 and its regulation by PUFA in colonic inflammation.

Infiltration of neutrophils into the site of inflammation is one of the hallmarks of $\mathrm{IBD}^{(50)}$. In the present study, colonic MPO was significantly increased in DSS-administered rats which were fed a diet with a high LA:ALA ratio, suggesting that neutrophils play an important role in DSS-induced colitis. However, decreasing the LA:ALA ratio to 2 significantly reduced colonic MPO activity and this was consistent with histological findings, DAI score and colon length.

Cytokines are potent inflammatory mediators and play a crucial role in inducing morphological changes in the colonic mucosa during inflammation ${ }^{(51)}$. Proinflammatory cytokines including TNF- $\alpha$ and IL- $1 \beta$ are elevated in patients with IBD and experimental models of colitis ${ }^{(52,53)}$. These proinflammatory cytokines cause neutrophil infiltration, tissue damage, ulceration and diarrhoea by disrupting the epithelial barrier function $^{(54)}$. Both ALA and LC $n-3$ PUFA have been shown to down-regulate the expression of proinflammatory genes through the inhibition of transcription factor $\mathrm{NF}-\mathrm{KB}^{(55)}$. Recently, Hassan et al. ${ }^{(42)}$ have demonstrated that dietary ALA supplementation reduces the expression of colonic TNF- $\alpha$ in trinitrobenzene sulphonic acid-induced colitis, thereby conferring protection. In the present study, TNF- $\alpha$ and IL-1 $\beta$ levels in the colon of DSS-administered rats which were fed a diet with a high LA:ALA ratio were significantly increased, suggesting that immune cells are attached to the site of inflammation. Substitution of LA with ALA suppressed inflammation by decreasing the production of proinflammatory cytokines. Increasing evidence indicates that colonic inflammation is associated with enhanced $\mathrm{NO}$ production, mainly via activation of inducible NO synthase activity $^{(56)}$. NO may potentiate the cytotoxicity by reacting with the superoxide anion to form peroxynitrite which is a potent pro-oxidant. A high intake of LA has been shown to cause nitrosative stress in the heart ${ }^{(57)}$. On the other hand, $n$-3 PUFA decrease NO production by down-regulating inducible NO synthase through the blocking of NF-кB activation $^{(58)}$. The data on colon NO levels in the present study reveal that DSS-induced colitis was associated with increased NO levels in the colon, while ALA supplementation markedly suppressed the NO production. These results suggest that ALA supplementation not only decreases proinflammatory cytokine levels but also reduces nitrosative stress, thereby offering protection against DSS-induced colitis.

Recent studies ${ }^{(59)}$ have documented that the gut microflora plays a pivotal role in the pathogenesis of human IBD and experimental colitis. Heimesaat et al. ${ }^{(60)}$ showed that DSS-induced colitis is associated with alteration in the gut microflora which resulted in a shift towards a proinflammatory Escherichia coli population. It is likely that the anti-inflammatory potential of ALA supplementation may be partly mediated through alteration in the composition and/or the type of gut microflora. Further studies are required to elucidate the link among dietary PUFA, gut microflora and DSS-induced colitis.

In conclusion, the results of the present study demonstrate that varying LA:ALA ratios, representing a wide range of 
LA:ALA intake in the present human diet, modulate colonic inflammation in the DSS-induced model of colitis. Further, the LA:ALA ratio of 2 was optimal for protection against colitis as evidenced by the reduction in neutrophil infiltration, preservation of colonic architecture and reversal of the shortening of colon length as well as improvements in the symptoms of colitis. These effects were associated with decreased production of proinflammatory mediators involved in the inflammatory response of the colon including cytokines such as TNF- $\alpha$, IL-1 $\beta$ and NO. The beneficial effects of ALA supplementation could be ascribed to the increased levels of LC $n-3$ PUFA and decreased levels of LC $n-6$ PUFA in the structural lipids of the colon. The results of this study hence reinforce the current recommendations for increasing $n-3$ PUFA in the diet for the prevention of diet-related chronic diseases.

\section{Acknowledgements}

The present project was funded by grants-in aid (SR/SO/ HS-001/2008) from the Department of Science and Technology, Government of India to A. I. A. T. was supported by a fellowship from Indian Council of Medical Research, Government of India. A. I. and A. T. designed the study and wrote the manuscript. U. K. carried out histological analysis of the colon. A. T., S. R., V. S. S. and S. B. M. conducted the animal experiments and the statistical analysis. N. Z. E. carried out cytokine estimation and contributed to the writing of the manuscript. All the authors contributed to data interpretation and approved the final version of the manuscript. None of the authors has any conflict of interest in relation to the present study.

\section{References}

1. Strober W, Fuss I \& Mannon P (2007) The fundamental basis of inflammatory bowel disease. J Clin Inves 117, 514-521.

2. Sartor RB (2006) Mechanisms of disease: pathogenesis of Crohn's disease and ulcerative colitis. Nat Clin Pract Gastroenterol Hepatol 3, 390-407.

3. Goh KL \& Xiao SD (2009) Inflammatory bowel disease: a survey of the epidemiology in Asia. J Digest Dis 10, 1-6.

4. Carr I \& Mayberry JF (1999) The effects of migration on ulcerative colitis: a three-year prospective study among Europeans and first- and second-generation South Asians in Leicester (1991-1994). Am J Gastroenterol 94, 2918-2922.

5. Wild GE, Drozdowski L, Tartaglia C, et al. (2007) Nutritional modulation of the inflammatory response in inflammatory bowel disease - from the molecular to the integrative to the clinical. World J Gastroenterol 13, 1-7.

6. Innis SM \& Jacobson K (2007) Dietary lipids in early development and intestinal inflammatory disease. Nutr Rev $\mathbf{6 5}$, S188-S193.

7. Schmitz G \& Ecker J (2008) The opposing effects of $n-3$ and n-6 fatty acids. Prog Lipid Res 47, 147-155.

8. Hulbert AJ, Turner N, Storlien LH, et al. (2005) Dietary fats and membrane function: implications for metabolism and disease. Biol Rev Camb Philos Soc 80, 155-169.

9. Simopoulos AP (2008) The importance of the omega-6/ omega-3 fatty acid ratio in cardiovascular disease and other chronic diseases. Exp Biol Med 233, 674-688.

10. Kang JX (2005) Balance of omega-6/omega-3 essential fatty acids is important for health. The evidence from gene transfer studies. World Rev Nutr Diet 95, 93-102.
11. John S, Luben R, Shrestha SS, et al. (2010) Dietary $n$-3 polyunsaturated fatty acids and the aetiology of ulcerative colitis: a UK prospective cohort study. Eur J Gastroenterol Hepatol 22, 602-606.

12. The IBD in EPIC Study Investigators (2009) Linoleic acid, a dietary $n-6$ polyunsaturated fatty acid, and the aetiology of ulcerative colitis: a nested case-control study within a European prospective cohort study. Gut 58, 1606-1611.

13. De Silva PS, Olsen A, Christensen J, et al. (2010) An association between dietary arachidonic acid, measured in adipose tissue, and ulcerative colitis. Gastroenterology 139, 1912-1917.

14. Belluzzi A, Boschi S, Brignola C, et al. (2000) Polyunsaturated fatty acids and inflammatory bowel disease. Am J Clin Nutr 71, 339S-342S.

15. Reeves PG, Nielsen FH \& Fahey GC (1993) AIN-93 purified diets for laboratory rodents: final report of the American Institute of Nutrition ad boc writing committee on the reformulation of the AIN-76A rodent diet. J Nutr 123, 1939-1951.

16. Ghafoorunissa, Ibrahim A \& Natarajan S (2005) Substituting dietary linoleic acid with alpha-linolenic acid improves insulin sensitivity in sucrose fed rats. Biochim Biophys Acta 1733, 67-95.

17. Ghafoorunissa, Reddy V \& Sesikaran B (1995) Palmolein and groundnut oil have comparable effects on blood lipids and platelet aggregation in healthy Indian subjects. Lipids 30, 1163-1169.

18. Gaudio E, Taddei G, Vetuschi A, et al. (1999) Dextran sulfate sodium (DSS) colitis in rats: clinical, structural, and ultrastructural aspects. Dig Dis Sci 44, 1458-1475.

19. Cooper HS, Murthy SN, Shah RS, et al. (1993) Clinicopathologic study of dextran sulfate sodium experimental murine colitis. Lab Invest 69, 238-249.

20. Krawisz JE, Sharon P \& Stenson WF (1984) Quantitative assay for acute intestinal inflammation based on myeloperoxidase activity. Assessment of inflammation in rat and hamster models. Gastroenterology 87, 1344-1350.

21. Dorai DT \& Bachhawat BK (1977) Purification and properties of brain alkaline phosphatase. J Neurochem 29 503-512.

22. Lowry $\mathrm{OH}$, Rosebrough NJ, Farr AL, et al. (1951) Protein measurement with the folin phenol reagent. $J$ Biol Chem 193, 265-275.

23. Folch J, Lees M \& Sloane Stanley GH (1957) A simple method for the isolation and purification of total lipides from animal tissues. J Biol Chem 226, 497-509.

24. Miranda KM, Espey MG \& Wink DA (2001) A rapid, simple spectrophotometric method for simultaneous detection of nitrate and nitrite. Nitric oxide 5, 62-71.

25. Ohkawa H, Ohishi N \& Yagi K (1979) Assay for lipid peroxides in animal tissues by thiobarbituric acid reaction. Anal Biochem 95, 351-358.

26. Vowinkel T, Mori M, Krieglstein CF, et al. (2004) Apolipoprotein A-IV inhibits experimental colitis. J Clin Invest $\mathbf{1 1 4}$ 260-269.

27. Kawada M, Arihiro A \& Mizoguchi E (2007) Insights from advances in research of chemically induced experimental models of human inflammatory bowel disease. World $J$ Gastroenterol 13, 5581-5593.

28. Nieto N, Fernandez MI, Torres MI, et al. (1998) Dietary monounsaturated, $n-3$ and $n-6$ long-chain polyunsaturated fatty acids affect cellular antioxidant defense system in rats with experimental ulcerative colitis induced by trinitrobenzene sulfonic acid. Dig Dis Sci 43, 2676-2687.

29. Nieto N, Torres MI, Rios A, et al. (2002) Dietary polyunsaturated fatty acids improve histological and biochemical 
alterations in rats with experimental ulcerative colitis. $J$ Nutr 132, 11-19.

30. Whiting CV, Bland PW \& Tarlton JF (2005) Dietary $n$-3 polyunsaturated fatty acids reduce disease and colonic proinflammatory cytokines in a mouse model of colitis. Inflamm Bowel Dis 11, 340-349.

31. Camuesco D, Galvez J, Nieto A, et al. (2005) Dietary olive oil supplemented with fish oil, rich in EPA and DHA (n-3) polyunsaturated fatty acids, attenuates colonic inflammation in rats with DSS-induced colitis. J Nutr 135, 687-694.

32. Hudert CA, Weylandt KH, Lu Y, et al. (2006) Transgenic mice rich in endogenous omega- 3 fatty acids are protected from colitis. Proc Natl Acad Sci U S A 103, 11276-11281.

33. Barceló-Coblijn G \& Murphy EJ (2009) Alpha-linolenic acid and its conversion to longer chain $n-3$ fatty acids: benefits for human health and a role in maintaining tissue $n-3$ fatty acid levels. Prog Lipid Res 48, 355-374.

34. Chan JK, McDonald BE, Gerrard JM, et al. (1993) Effect of dietary alpha-linolenic acid and its ratio to linoleic acid on platelet and plasma fatty acids and thrombogenesis. Lipids 28, 811-817.

35. Fu Z \& Sinclair AJ (2000) Increased alpha-linolenic acid intake increases tissue alpha-linolenic acid content and apparent oxidation with little effect on tissue docosahexaenoic acid in the guinea pig. Lipids 35, 395-400.

36. Aziz AA, Cruz-Hernandez C, Plouffe LJ, et al. (2010) Increasing dietary alpha-linolenic acid enhances tissue levels of long-chain $n$-3 PUFA when linoleic acid intake is low in hamsters. Ann Nutr Metab 57, 50-58.

37. Goyens PL, Spilker ME, Zock PL, et al. (2006) Conversion of alpha-linolenic acid in humans is influenced by the absolute amounts of alpha-linolenic acid and linoleic acid in the diet and not by their ratio. Am J Clin Nutr 84, 44-53.

38. Heimerl S, Moehle C, Zahn A, et al. (2006) Alterations in intestinal fatty acid metabolism in inflammatory bowel disease. Biochim Biophys Acta 1762, 341-350.

39. Tu WC, Cook-Johnson RJ, James MJ, et al. (2010) Omega-3 long chain fatty acid synthesis is regulated more by substrate levels than gene expression. Prostaglandins Leukot Essent Fatty Acids 83, 61-68.

40. Blank C, Neumann MA, Makrides M, et al. (2002) Optimizing DHA levels in piglets by lowering the linoleic acid to alphalinolenic acid ratio. J Lipid Res 43, 1537-1543.

41. Calder PC (2008) Polyunsaturated fatty acids, inflammatory processes and inflammatory bowel diseases. Mol Nutr Food Res 52, 885-897.

42. Hassan A, Ibrahim A, Mbodji K, et al. (2010) An $\alpha$-linolenic acid-rich formula reduces oxidative stress and inflammation by regulating NF- $\mathrm{\kappa B}$ in rats with TNBS-induced colitis. J Nutr 140, 1714-1721.

43. Baumeister B, Schmidt C, Helisch A, et al. (1996) Increased prostaglandin $\mathrm{E}_{2}$ and leukotriene $\mathrm{B}_{4}$ synthesis in isolated colonic mucosal cells in inflammatory bowel disease. a preliminary report. J Clin Gastroenterol 22, 117-120.

44. Levy BD (2010) Resolvins and protectins: natural pharmacophores for resolution biology. Prostaglandins Leukot Essent Fatty Acids 82, 327-332.

45. Ishida $T$, Yoshida $M$, Arita $M$, et al. (2010) Resolvin $E_{1}$, an endogenous lipid mediator derived from eicosapentaenoic acid, prevents dextran sulfate sodium-induced colitis. Inflamm Bowel Dis 16, 87-95.

46. Miyazaki M, Man WC \& Ntambi JM (2001) Targeted disruption of stearoyl-CoA desaturase1 gene in mice causes atrophy of sebaceous and meibomian glands and depletion of wax esters in the eyelid. J Nutr 131, 2260-2268.

47. MacDonald ML, van Eck M, Hildebrand RB, et al. (2009) Despite antiatherogenic metabolic characteristics, SCD1deficient mice have increased inflammation and atherosclerosis. Arterioscler Thromb Vasc Biol 29, 341-347.

48. Chen C, Shah YM, Morimura K, et al. (2008) Metabolomics reveals that hepatic stearoyl-CoA desaturase 1 downregulation exacerbates inflammation and acute colitis. Cell Metab 7, $135-147$

49. Macdonald ML, Bissada N, Vallance BA, et al. (2009) Absence of stearoyl-CoA desaturase-1 does not promote DSS-induced acute colitis. Biochim Biophys Acta 1791, 1166-1172.

50. Domek MJ, Iwata F, Blackman EI, et al. (1995) Antineutrophil serum attenuates dextran sulfate sodium-induced colonic damage in the rat. Scand J Gastroenterol 30, 1089-1094.

51. Sanchez-Munoz F, Dominguez-Lopez A \& YamamotoFurusho JK (2008) Role of cytokines in inflammatory bowel disease. World J Gastroenterol 14, 4280-4288.

52. Reinecker HC, Steffen M, Witthoeft T, et al. (1993) Enhanced secretion of tumour necrosis factor-alpha, IL-6, and IL-1 beta by isolated lamina propria mononuclear cells from patients with ulcerative colitis and Crohn's disease. Clin Exp Immunol 94, 174-181.

53. Egger B, Bajaj-Elliott M, MacDonald TT, et al. (2000) Characterization of acute murine dextran sodium sulphate colitis: cytokine profile and dose dependency. Digestion 62, 240-248.

54. McGuckin MA, Eri R, Simms LA, et al. (2009) Intestinal barrier dysfunction in inflammatory bowel diseases. Inflamm Bowel Dis 15, 100-113.

55. Calder PC (2002) Dietary modification of inflammation with lipids. Proc Nutr Soc 61, 345-358.

56. Pavlick KP, Laroux FS, Fuseler J, et al. (2002) Role of reactive metabolites of oxygen and nitrogen in inflammatory bowel disease. Free Radic Biol Med 33, 311-322.

57. Ghosh S, Novak EM \& Innis SM (2007) Cardiac proinflammatory pathways are altered with different dietary $n$-6 linoleic to $n-3$ alpha-linolenic acid ratios in normal, fat-fed pigs. Am J Physiol Heart Circ Physiol 293, H2919-H2927.

58. Ren J \& Chung SH (2007) Anti-inflammatory effect of alpha-linolenic acid and its mode of action through the inhibition of nitric oxide production and inducible nitric oxide synthase gene expression via NF-kappaB and mitogen-activated protein kinase pathways. J Agric Food Chem 55, 5073-5080.

59. Fava F \& Danese S (2011) Intestinal microbiota in inflammatory bowel disease: friend of foe? World J Gastroenterol $\mathbf{1 7}, 557-566$

60. Heimesaat MM, Fischer A, Siegmund B, et al. (2007) Shift towards pro-inflammatory intestinal bacteria aggravates acute murine colitis via Toll-like receptors 2 and 4. PLOS One 2, e662. 